\title{
XLVI,
}

\section{Antwort und Selbstvertheidigung gegenüber den Angriffen des Herrn Prof. Erb.}

Von

\author{
Dr. Andreas Takács.
}

\begin{abstract}
Auf Grund des Inhalts meiner Mittheilung über "Die graue Degeneration der hinteren Rüekenmarksstränge und die Ataxie" (dieses Archiv IX. Bd. letztes Heft), hat mich Herr Prof. Erb auf das Heftigste angegriffen (dieses Archir X. Bd. 1. Heft).
\end{abstract}

Das Hauptmotiv seines Angriffes bildet mein Auspruch: er habe die locomotorische Ataxie von der Sensibilität unabhängig, dagegen die statische Ataxie von derselben abhängig erklärt.

Diese meine Behauptung ist in der That irrthümlich, und wird einerseits dadurch begründet, dass Herr Prof. Erb in seinen: "Krankheiten des Rückenmarks" (1. Abth. "1876" Seite 40) lediglich von Bewegung spricht, welche ich mit der Friedreich'schen locomotorischen Coordination identificirte (Virch ow's Archiv 68. Band "1876"); anderseits verstand ich unter „Erhaltung des Gleichgewichtes" auch das Stehen und Sitzen, welche Friedreich in die Reihe der Erscheinungen von statischer Ataxie zählt.

Wenn ich jedoch diesen Fehler anerkenne, so sei damit jene meine Behauptung noch nicht aufgegeben: dass Herr Prof. Erb mit der Sensibilität willkürlich und sich widersprechend vorgeht.

Nach Herrn Prof. Erb ist jede coordinirte Bewegung (mit Ausnahme der angeborenen) Resultat des Erlernens, bei welchem die Sensibilität eine sehr wichtige Rolle spiele (1. Abtheil. S. 39); ferner handle es sich bei "Erhaltung des Gleichgewichts des Körpers" um foin coordinirte Muskelcontractionen (ibid. S. 41). Die einmal angeeignete coordinirte Bewegung sei aber - nach ihm — von der Sensibilität vollkommen unabhängig; denn: „das lässt sich daran zeigen, dass wir . . . . . die complicirtesten Bewegungen mit einer Raschheit und Sicherheit ausführen 
können, welche jeden Gedanken an eine..... sensorische Controle ausschliesst". (ibid. S. 40.) Später behauptet er: es sei sowohl die locomotorische wie auch statische Coordination von der Sensibilität unabhängig (2. Abth. S. 169). - Hingegen sagt er von der - ebenfalls a u coordinirte Mukelthätigkeit basirenden - Erhaltung desGleichgewichts: dieselbe hänge stets und immer von der Sensibilität ab. (1. Abth. S. 41.) -

Nach ihm soll - beim Erlernen allein - die Art und Weise der coordinirten Bewegung im Centrum des Willens durch die Sensibilität angezeigt werden, damit der Wille die nöthigen Modificationen in den Coordinationscentren einleite. Bei der Erhaltung des Gleichgewiehts hingegen, soll statt des Centrums des Willens ein Gleichgewichts-Centrum fungiren, welches die Sensibilität beständig - also auch naoh dem Erlernen - beeinflussen sollte.

Dieser Auffassung gegenüber halte ich den Einfluss der Sensibilität in den Coordinationscentren - auch nach dem Erlernen - für beständig, und zwar ohne Vermittelung eines anderen Centrums, bei allen coordinirten Muskelthätigkeitserscheinungen ( $\mathrm{L} \dot{\theta} \mathrm{y} d \mathrm{en}, \mathrm{Clark} \theta)$.

Nach dem Gesagten erkläre ich also : dass ich darin einen noch grösseren Widerspruch erblicke, wenn Herr Prof. Erb behauptet, die locomotorische und statische Coordination sei von der Sensibilität unabhängig, hingegen die Aufrechterhaltung des Gleichgewichts von derselben abhängig.

Wenn die Sensibilität bei der Aneignung verschiedener Formen der coordinirten Muskelthätigkeiten und bei der Erhaltung des Gleichgewichts eine so hervorragende Rolle spielt, so ist es schwer einzusehen, warum sio bei der einen als Controle beständig verbleibt, während sie für die andere überflüssig wird.

Die Einwendung, dass manche Bewegung allzu rasch vor sich geht (z. B. Sprung, Wurf u. s. w.) ist nicht von Belang; denn hinsichtlich der verflossenen Zeit ist der Unterschied bei allen coordinirten Bewegungen nur ein gradueller, und ein solcher vermag in der Natur liein Gesetz zu stürzen. Was für eine coordinirte Bewegung giltig, ist es auch für die andere. - Wenn es wirklich richtig ist, dass die Sensibilität beim Erlernen der coordinirten Bewegungen und der Aufrechterhaltung des Gleichgewichts einen so hochwichtigen Factor bildet - woron ich überzeugt bin, and was auch von Herrn Prof. Erb angenommen wird - dann ist es gewiss ein eigenmächtiges Vorgehen, den Sensibilitätseinfluss bei der locomotorischen und statischen Ataxie nicht gelten lassen zu wollen.

Es folge nun auch der Grund desselben.

Während Herr Prof, Erb die locomotorische und statische Ataxie als constantes, und von der Sensibilität unabhängiges Symptom der Tabes bezeichnet, hält er die Störung der Erhaltung des Gleichgewichtes anscheinend für ein blos begleitendes Symptom, und hält es nur dann für vorhanden, wenn der mit Sensibilitätsstörung behaftete Kranke beim Schluss der Augen schwankt (ibid. S. 92). Er betrachtet sogar dieses Brach-Romberg'sche 
Symptom als ein Zeichen noch nicht nachweisbarer Sensibilitätsstörung (1. Abtheil. S. 174).

Herr Prof. Erb geht also mit der Sensibilität bei pathologischen Erscheinungen ebenso vor, wie ich es bei normal-coordinirter Muskelthätigkeit beschrieb. Nach ihm ist die locomotorische und statische Ataxie von der Sensibilitätsstörung unabhängig (ibid. S. 169), dagegen erscheint die Störung der Erhaltung des Gleichgewichts lediglich der Sensibilitäts-Abnahme untergeordnet (ibid. S. 174).

Wollte ich diesen Widerspruch einer Kritik unterwerfen, so müsste ich in eine Wiederholung des oben Gesagten verfallen. Es sei somit nur hervorgehoben, dass Herr Prof. Erb in seinem Lehrbuch (1. Abth. S 93) die Steigerung der Ataxie — bei Schluss der Augen - wie folgt erklärt: „dass also-ähnlich wie beim Erlernen der Coordination ein beständiger Einfluss auf die Coordinationscentren hergestellt werden kann!“ - Also die Sensibilität obwohl hier nur vom Gesichtssinn die Rede ist - übt dennoch ihre Wirksamkeit auf die Ataxie, wenn Herr Prof. Erb die Steigerung derselben - lauteigener motorischer Theorie - zu erklären nicht mehr im Stande ist!!

Ich beabsichtige jedoch meine Erwiederung durch ähnliche Cita te nicht übermässig auszudehnen; deshalb möchte.ich nur noch auf jene Nothwendigkeit hindeuten, vermöge welcher Herr Prof. Erb den Einfluss der Sensibilität hie und da zugelassen hat.

Behufs Erklärung der atactischen Muskelthätigkeit stellte $\theta \mathrm{r}$ eine Theorie auf, welche, wenn dèr Grund des Leidens nicht in der Degeneration der hinteren, - centripetale Fasern enthaltenden - Rückenmarkstränge zu suchen wäre, eine jede derartige Muskelthätigkeit, aus-genommen die Steigerung der Ataxie beim Schlus der Augen, aufzuklären thatsächlich (?) berufen sein könnte! Denn wie so liesse sich mit Zuhilfenahme einer Theorie, - "motorisohe Ataxie" genannt —, welche die Ataxie von Erkrankung der centrifugalen Nervenfasern herleitet, eine solche Muskelthätigkeits-Incoordination in's klare Lichtstellen, welche nur beim Schluss der Augen eintritt.

Wäre die Incoordination durch Erkrankung centrifugaler - motorischcoordinatorischer - Nervenfasern bedingt (wofür jedoch bis jetzt jedwede anatomische, physiologische undpathologische Begründung mangelt), dann müsste das Schwanken sowohl bei offenen wie bei geschlossenen Augen auftreten; oder wenigstens dürfte sich die Ataxie bei geschlossenen Augen nicht steigern. Wenn sich aberdas Schwanken bei geschlossenen Augen einstellt und die Ataxiesich erhöht, müsste nothwendigerweise zur Sensibilität gegriffen wer. den, welche zuvor durch den Gesichtssinnsubstituirt war!

Hierin die Ursache, weshalb die Sensibilität bei der Erhaltung des Gleichgewichts und bei der Ataxie - wenn letztere beim Schluss der Augen zunimmt - eine Rolle spielt.

Während Herr Prof. Erb, die Schwerfälligkeit dieses Vorgehens mit 
der Sensibilität fühlend, die Ërrogung der sensiblen Fasern - bei locomotorischer und statischer Coordination (nach Erlernen) - nebst Vermittlung des Willenscentrums nicht zu den Coordinationseentren führt, leitet er die Erregung der sensiblen Nerven - bei Erhaltung des Gleichgewichts (immerwährend) - durch die Gleichgewichtscentren zu den Coordinationscentren, welche sodann die erforderliche Correction der Muskelthätigkeit bewirken.

Laut dem Angeführten sollten also einerseits jene sensiblen Bahnen, vermittelst welcher die durch Muskelthätigkeit ausgelösten Reize zu den Coordinationscentren gelangten, -- nach erlernten Bewegungen - zu Grunde gehen, um erst dann wieder zu entstehen, wennsiedie Steigerung der Ataxie bei geschlossenen Augen zu begründen hätten; andererseits müsste eine Art der coordinirten Bewegungen (nämlich die Erhaltung des Gleichgewichts) die sensiblen Nerven ausschliesslich in Anspruch nehmen, sogar für sich separate Gleichgewichtscentren bilden, um sich dadurch dem Einfluss der Gesetze sämmtlicher coordinirten Muskeltbätigkeiten za entziehen!

Oder beging ich denn einen Fehler, als ich diese Naturgesetzwidrigkeit der Erb'schen Theorie heraus fand, und nach einer anderen Theorie strebte, aus welcher auch die Schwankung beim Schluss der Augen hergeleitet werden kann?

Als Antwort hege ich die feste Ueberzeugung, dass meine sensorische - auf Sensibilitäts-Leitungs - Verspätung basirte Theorie, - welche sich auf Schiff's zwar alte, doch bis jetzi nicht verworfene physiologische Experimente, auf Flechsig's anatomische Untersuchungen, auf Remak's, Naunyn's und Burghardt's Beobachtungen, endlich auf fremde und eigene Erfahrungen stützt, die jede feste Basis entbehrende "motorische A taxie" überle ben wird, wenn letztere sich auf Erkrankung des Rückenmarkes bezieht.

Es sei mir boch gestattet, darauf hinzuweisen, dass Herr Prof. Erb das Wesen meiner Arbeit - den Krankheitsfall und die daraus gezogenen Folgerungen - ohne Bemerkung liess; dass er die Ausdrücke: ,motorische" und "Ruhezustände" — mit einigem Wohlwollen - um so mehr für Druckfehler hätte ansehen können, als ich dieselben genau umschrieb; ferner dass sich meine Theorie in die Reihe der sensorischen Theorien stellt, mit deren Umstürzung - falls die motoris che Theorie eine jetzt noch mangelnde, aber sehr nothwendige Basis erlangt, - auch sie ihre Geltung verliert, dass sie heute jedoch mehr anatomische und physiologische Grundlage besitzt, als jene motorische Theorie.

Sollte endlich Herr Prof. Erb einer eventuell ernenerten Anfechtung den gleichen Ton zu Grunde legen, so möchte ich ihm darin nichtsdestoweniger weder nachahmen können noch wollen, somit auf eine Antwort vollends verzichten.

Budapest, im Januar 1880. 